\title{
CONF-970503--19 JLAB-ACC-97-19 MEASURING LONGITUDINAL DISTRIBUTION AND BUNCH LENGTH OF FEMTOSECOND BUNCHES WITH RF DOE/ER/40150--1/22 ZERO-PHASING METHOD
}

\author{
D. X. Wang, and G. A. Krafft, Thomas Jefferson National Accelerator Facility \\ 12000 Jefferson Avenue, Newport News, VA
}

\begin{abstract}
Recently there has been increasing interest in applications of very short electron bunches. Accurately measuring bunch length and profiles becomes essential for characterizing, commissioning, and operating such short bunch machines. The RF zero-phasing method is the only technique that is able to measure bunch length and longitudinal density distribution in the femtosecond regime. In this paper, analytical formulas for calculating longitudinal profile and bunch length are given. Using a such technique, bunch lengths as short as $84 \mathrm{fs}$ rms have been measured.
\end{abstract}

\section{INTRODUCTION}

In recent years, there has been increasing interest in the use of very short electron bunches [1-3]. The shortest bunch for proposed and existing accelerators is around 100 fs (rms). Bunch length measurement is essential to develop and operate such accelerators. Such measurement also plays a crucial role in developing and advancing new techniques such as the coherent radiation method [4].

It becomes very challenging for conventional techniques such as streak cameras to resolve bunch lengths of a few hundred femtoseconds [5]. Recently developed frequency domain techniques measuring coherent radiation spectra are not able to uniquely determine bunch shape and length $[4,6-9]$. The RF zerophasing technique has been used to measure longitudinal distributions and bunch lengths of picosecond bunches. Good consistency was found between measurement and simulations [10-14]. However, the longitudinal profiles reported in Ref. 10 and 12 were obtained where the transverse beam sizes can be neglected, while the formula used in Ref. 11 to calculate bunch lengths is only valid for Gaussian distributions. There is no general method to determine bunch shape and length. Furthermore, it is not clear if this technique can be applied to the femtosecond regime. In this paper, analytical formulas are given for computing the longitudinal distribution function and bunch length for arbitrary longitudinal and transverse distributions. Applicability of the technique to femtosecond bunches is demonstrated and confirmed by numerical simulations.

\section{RF ZERO-PHASING METHOD}

The zero-phasing measurement utilizes several RF accelerating cavities (the zero-phasing cavities), a spectrometer, and a horizontal profile measuring device." The zero-phasing cavities operate at the zero-crossingf the accelerating wave and impart a time-correlarte momentum deviation along the beam bunch. Then a spectrometer translates the longitudinal momentum spread into a horizontal position spread. Based on measurement of the horizontal profiles, the bunch length and longitudinal distribution function can be determined.

With the following two assumptions, an analytical expression for the longitudinal distribution function can be derived as a function of the horizontal distribution function and horizontal profile measured at the spectrometer with the zero-phasing cavities on. First, it is assumed that the distribution function in the longitudinal and transverse directions can be separated, i.e.

$$
f\left(x, x^{\prime}, z, z^{\prime}\right)=f_{x}\left(x, x^{\prime}\right) f_{z}\left(z, z^{\prime}\right),
$$

where $f, f_{x}$, and $f_{z}$ are the total, longitudinal, and horizontal normalized distribution functions, respectively. Second, it is assumed that the longitudinal phase space can be treated as an ellipse, and that the intrinsic energy spread or the width of the ellipse can be neglected.

The horizontal position of an electron at the spectrometer can be then written as $X=x+C z$, where $x$ is the nondispersive part,

$$
\begin{aligned}
C & =C_{0}+C_{1} \\
& =\left(2 \pi \cdot e V_{\mathrm{rf}} / \lambda_{\mathrm{rf}}+d E / d z\right) \cdot \eta / E_{0},
\end{aligned}
$$

$\eta$ is dispersion of the spectrometer, $E_{0}$ is average beam energy, $\lambda_{\mathrm{rf}}$ is the RF wavelength, $V_{\mathrm{rf}}$ is the summed accelerating voltage of the zero-phasing cavities, and $d E / d z$ is the slope of the longitudinal phase space ellipse. The linear slope of the accelerating voltage of the zero-phasing cavities, i.e. $2 \pi \cdot e V_{\mathrm{rf}} / \lambda_{\mathrm{rf}}$, is used under the approximation that the $R F$ wavelength is much longer than the bunch length. The horizontal profile measured at the spectrometer with the zero-phasing cavities on is then found to be

$$
\begin{aligned}
& F(x)=\iiint f_{x}\left(x, x^{\prime}\right) d x^{\prime} f_{z}\left(z, z^{\prime}\right) d z d z^{\prime} \\
& \quad=\int f_{x}(x+C z) f_{z}(z) d z,
\end{aligned}
$$

where $\int f_{x}\left(x, x^{\prime}\right) d x^{\prime}=f_{x}(x)$ and $\int f_{z}\left(z, z^{\prime}\right) d z^{\prime}=f_{z}(z)$ are used by definition. Therefore, the measured horizontal profile is simply a convolution of the longitudinal distribution function and the horizontal distribution function. The longitudinal distribution function can be deconvolved as 


\section{DISCLAIMER}

This report was prepared as an account of work sponsored by an agency of the United States Government. Neither the United States Government nor any agency thereof, nor any of their employees, make any warranty, express or implied, or assumes any legal liability or responsibility for the accuracy, completeness, or usefulness of any information, apparatus, product, or process disclosed, or represents that its use would not infringe privately owned rights. Reference herein to any specific commercial product, process, or service by trade name, trademark, manufacturer, or otherwise does not necessarily constitute or imply its endorsement, recommendation, or favoring by the United States Government or any agency thereof. The views and opinions of authors expressed herein do not necessarily state or reflect those of the United States Government or any agency thereof. 


\section{DISCLAMMER}

Portions of this document may be illegible in electronic image products. Images are produced from the best available original document. 


$$
f_{z}(z)=\frac{C}{2 \pi} \int\left[\frac{\int F(x) e^{i k x} d x}{\int f_{x}(x) e^{i k x} d x}\right] e^{i c z k} d k .
$$

Now $f_{x}(x)$ may be directly measured or it may be approximated by the horizontal profile measured at the spectrometer with the zero-phasing cavities off, if the dispersive part of the initial energy spread is small compared to the horizontal beam size. Such an approximation was made for the following measurement. It can be shown from Eq. (4) that if the horizontal distribution function and the measured horizontal profiles are Gaussian, the longitudinal distribution function is also Gaussian. The longitudinal bunch length is given to

$$
\sigma_{z}=\left(\Omega^{2}-\sigma_{x}^{2}\right)^{1 / 2} / C,
$$

where $\sigma_{z}, \Omega$, and $\sigma_{x}$ are corresponding widths of the Gaussian profiles, as used in Ref. 11. If $f_{x}(x)$ is negligibly small compared to $F(x)$ and can be replaced by a delta function, the longitudinal distribution function equals the measured profile, i.e. $f_{z}(z=x / C)=F(x) / C$ as used in ref. 10 and 12 , with a scale conversion factor $C$ between the horizontal and longitudinal dimensions. Usually, $C_{0}$ can be experimentally determined, but $d E / d z$ or $C_{1}$ is unknown. To overcome this problem, the horizontal profiles are measured at both zero-crossing points of the RF waves, which alters the sign of $C_{0}$. It is noticed that using $C_{0}$ instead of $C$ in $\mathrm{Eq}$. (4) is equivalent to compressing the $Z$ axis by a factor of $C / C_{0}$. Therefore, the longitudinal $\mathrm{ms}$ length is

$$
\begin{aligned}
z_{\mathrm{rms}} & =z_{\mathrm{rms}}^{+} \cdot C_{0} /\left(C_{0}+C_{1}\right) \\
& =z_{\mathrm{ms}}^{-} \cdot C_{0} /\left(C_{0}-C_{1}\right),
\end{aligned}
$$

where $z_{\mathrm{ms}}^{+}$and $z_{\mathrm{ms}}^{-}$are the rms lengths with the zerophasing cavity at $+90^{\circ}$ and $-90^{\circ}$ off crest, respectively, using $C_{0}$. Then the rms length and initial phase space slope normalized by the RF slope are given by

$$
z_{\mathrm{ms}}=\left(z_{\mathrm{ms}}^{+}+z_{\mathrm{ms}}^{-}\right) / 2
$$

and

$$
C_{1} / C_{0}=(d E / d z) /\left(2 \pi \cdot e V_{\mathrm{rr}} / \lambda_{\mathrm{rf}}\right)
$$

$$
=\left(z_{\text {rms }}^{+}-z_{\text {rms }}\right) / z_{\text {rms }} \text {. }
$$

Accordingly, the $z$ axis for both measured profiles needs to be corrected by the corresponding factor of $C_{0} / C$. The difference between the two resulting distribution functions indicates the validation of the assumptions.

\section{MEASUREMENT AND SIMULATION RESULTS}

The formula and measurement procedure were tested using PARMELA simulation, which has been used extensively during CEBAF commissioning to verify experimental results. Very good agreement has been found between various measurements and simulation results in the past. The zero-phasing measurement was performed numerically and compared to the actual bunch length in the simulation. The test case showed good agreement over bunch lengths in the range of 100 to $400 \mathrm{fs}$, with a $10 \mathrm{fs}$ systematic offset, indicating that the assumptions are good approximations.

The measurement was carried out at the CEBAF injector [15-16]. A $500 \mathrm{keV}$ bunched electron beam is further bunched and accelerated to $5 \mathrm{MeV}$ by the two superconducting RF (SRF) cavities. Following acceleration to the final injection energy of $45 \mathrm{MeV}$ by 16 SRF cavities, little additional bunching occurs, due to the relativistic effects. Nominally, 16 SRF cavities run on crest. During the measurement of the shortest bunches, the last 8 SRF cavities are phased to plus and minus $90^{\circ}$ off crest. A wire scanner is used to measure the horizontal profile at the spectrometer. Three typical profiles are shown in Fig. 1 and can be fit closely to Gaussian distributions. Both zero phases of each individual zerophasing cavity are determined by finding the phase that yields zero transverse movement at the spectrometer viewer when the cavity is turned on and off. The beam energy and summation of the zero-phasing cavity gradient were measured by the spectrometer Hall probe. The experimental parameters in this measurement are: $\lambda_{\mathrm{rf}}$ of $20 \mathrm{~cm}, \eta$ of $1.52 \mathrm{~m}, V_{\mathrm{rf}}$ of about $20 \mathrm{MV}$, and $E_{0}$ of
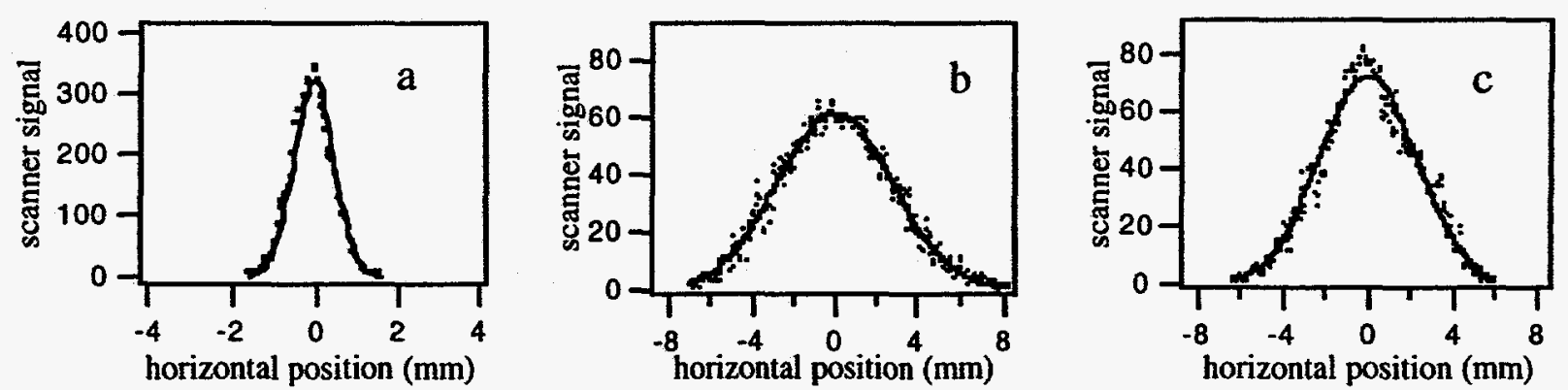

Fig. 1 Horizontal profile measured by the wire scanner at the spectrometer, where the scanner signal is proportional to the charge density and the circles are from measurement and the solid curves are Gaussian fittings. Profile (a) was measured with the zero-phasing cavities off while profiles (b) and (c) were measured with the zero-phasing cavities at $+90^{\circ}$ and $-90^{\circ}$ off the crest, respectively. 
about $25 \mathrm{MeV}$. The bunch length was systematically changed by varying the second SRF cavity phase, resulting in a series of longitudinal phase space rotations. Excellent agreement has been achieved between the measurement and simulation, shown in Fig. 2. In addition, the measurement results are consistent with a power measurement of the coherent synchrotron radiation (CSR) [17]. As expected, CSR power increased when the bunch length became shorter, and minimum bunch length yielded maximum CSR power. The left side of Eq. (8) is plotted from simulation in the solid line while the right side is displayed from measurement in the circles of Fig. 3 , as the phase of the second SRF bunching cavity is varied as in Fig. 2. Measurements and simulation results agree well. It is noted that the zero value point represents the upright position of the ellipse in the longitudinal phase space, where the shortest bunch was obtained in both experiment and simulation, i.e. the minimum point in Fig. 3. There is a steep slope around the zero point where the slope of the ellipse changes sign, corresponding to the transition from under-compression to overcompression. Therefore, the maximum slope of the ellipse $d E / d z$ is about a factor of seven smaller than the RF slope, $2 \pi \cdot e V_{\mathrm{rf}} / \lambda_{\mathrm{rf}}$ of $720 \mathrm{MeV} / \mathrm{m}$.

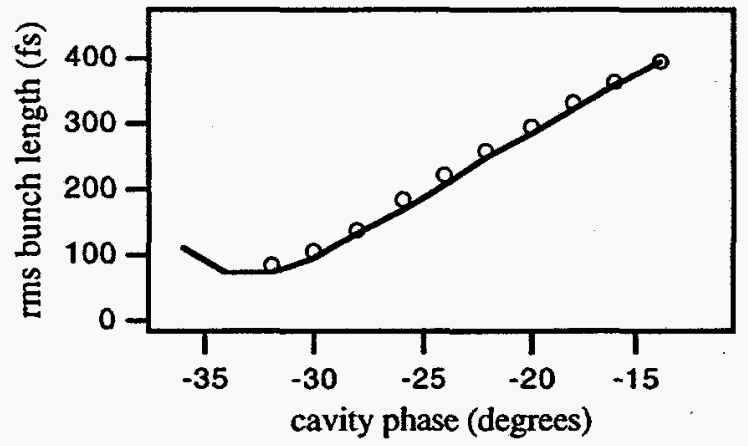

Fig. 2 The bunch length versus the second SRF cavity phase, where the circles are from measurement and the solid curve is from simulation.

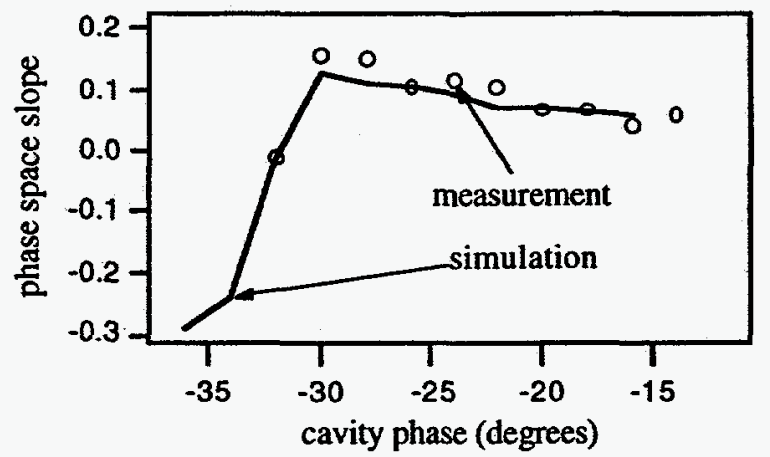

Fig. 3 The normalized longitudinal phase space slopes. The circles are the measurement of the right-hand side of Eq. 8, $\left(z_{\text {ms }}^{+}-z_{\text {ms }}\right) / z_{\text {ms }}$, while the solid curve is the left hand side, $(d E / d z) /\left(2 \pi \cdot e V_{\mathrm{r}} / \lambda_{\mathrm{r}}\right)$, from simulation.

The zero-phasing technique provides a means of measuring the longitudinal density distribution function and the bunch length of femtosecond bunches. Its resolution is mainly limited by the constraints of the specific machine configuration. In practice the following considerations may be given to better interpret the measurement results: (1) measure the horizontal distribution function at the straight-ahead location and the horizontal profile at the spectrometer with the zerophasing cavities off, which provides information about the longitudinal phase space ellipse, with orientation information obtained from Eq. (8); (2) focus the beam spot tightly in the horizontal direction; (3) increase the energy spread produced by the zero-phasing cavities; (4) the errors due to RF amplitude and phase stability can be estimated from horizontal beam jitters at the spectrometer viewer, which result in a longer measured bunch length. The zero-phasing technique has been proven to be crucial at CEBAF for characterizing the bunching process and calibrating a noninvasive CSR bunch length monitor that is an invaluable tool for machine operations.

\section{CONCLUSION}

In summary, analytical formulas for calculating the longitudinal distribution function and bunch length are derived. An electron bunch length as short as $84 \mathrm{fs}$ (rms) has been measured. This is the first accurate bunch length measurement in the regime of less than $100 \mathrm{fs}$, and the zero-phasing is the only technique that has demonstrated such a capability. Numerical simulation provides validation of the assumptions of the technique. The systematic measurement results are in excellent agreement with the simulation and consistent with CSR power measurements.

\section{ACKNOWLEDGMENT}

The authors acknowledge C. K. Sinclair for his guidance and support, and also thank B. Dunham and H. Liu for help running the PARMELA code and CEBAF operations personnel for their help. This work was supported by U.S. DOE under contract number DE-AC05-84ER40150.

\section{REFERENCES}

[1] H. Winick, et al., NIM, A347 (1994), 199.

[2] R. H. Siemann, Proc. of 1993 PAC Conf., 532.

[3] W. B. Mori, et at., AIP Conf. Proc. 335, 112 (1994).

[4] D. X. Wang, "Electron Beam Instrumentation Techniques Using Coherent Radiation", these proceedings.

[5] A. H. Lumpkin, AIP Conf. 367, 327 (1995).

[6] K. Ishi, et al., Phys. Rev. A43, 5597 (1991).

[7] Y. Shibata, et al., Phys. Rev. E50, 1479 (1994).

[8] R. Lai, et al., Phys. Rev. E 52, 6787 (1995).

[9] H. C. Lihn, et al., Phys. Rev. E53, 6413 (1996).

[10] L. Rivkin et al., Proc. of 1st EPAC (1988), 634.

[11] J. Seeman et al., Proc. of 1988 LINAC Conf., 441.

[12] K. Bane, SLAC-PUB-5177, Feb. 1990 (A).

[13] K. Bane et al., Proc. of 2nd EPAC (1990), 1762.

[14] K. Bane et al., SLAC/AP-80, Sept. 1990 (AP).

[15] G. A. Krafft, Proc. of 1994 LINAC Conf., 9.

[16] D. X. Wang, Proc. of 1996 LINAC Conf., 303.

[17] D. X. Wang, et al., Appl. Phys. Lett. 70 (4), 529 (1997). 